\title{
Publisher Correction: Open is not enough
}

Xiaoli Chen, Sünje Dallmeier-Tiessen, Robin Dasler, Sebastian Feger, Pamfilos Fokianos, Jose Benito Gonzalez, Harri Hirvonsalo, Dinos Kousidis, Artemis Lavasa, Salvatore Mele, Diego Rodriguez Rodriguez, Tibor Šimko, Tim Smith, Ana Trisovic, Anna Trzcinska, loannis Tsanaktsidis, Markus Zimmermann, Kyle Cranmer, Lukas Heinrich, Gordon Watts, Michael Hildreth, Lara Lloret Iglesias, Kati Lassila-Perini and Sebastian Neubert

Correction to: Nature Physics https://doi.org/10.1038/s41567-018-0342-2 published online 15 November 2018.

In the version of this Perspective originally published, one of the authors' names was incorrectly given as Kati Lassili-Perini; it should have been Kati Lassila-Perini. This has been corrected in all versions of the Perspective.

Published online: 23 November 2018

https://doi.org/10.1038/s41567-018-0382-7

\section{Publisher Correction: Spin transport and spin torque in antiferromagnetic devices}

\author{
J. Železný, P. Wadley, K. Olejník, A. Hoffmann and H. Ohno
}

Correction to: Nature Physics https://doi.org/10.1038/s41567-018-0062-7, published online 2 March 2018.

In the version of this Review Article originally published, the affiliations of the authors J. Železný, P. Wadley and K. Olejník were incorrect and should have read: "J. Železný'1,2, P. Wadley ${ }^{3}$, K. Olejník ${ }^{2} .{ }^{1}$ Max Planck Institute for Chemical Physics of Solids, Dresden, Germany. ${ }^{2}$ Institute of Physics, Academy of Sciences of the Czech Republic, Praha, Czech Republic. ${ }^{3}$ School of Physics and Astronomy, University of Nottingham, Nottingham, UK." These have now been corrected in the online versions.

Published online: 18 December 2018

https://doi.org/10.1038/s41567-018-0402-7

\section{Publisher Correction: High-harmonic generation from solids}

Shambhu Ghimire and David A. Reis

Correction to: Nature Physics https://doi.org/10.1038/s41567-018-0315-5, published online 19 November 2018.

In the version of this Review Article originally published, in Fig. 2b, the label 'Inter-band current' should have read 'Intra-band current'. This error has now been corrected in the online versions. 